\title{
Predicting China's Aging Portfolio Model Based on Python Big Data
}

\author{
Buyu DONG, Mingjun ZU, Youhui SU1 and Xucheng DING \\ College of Mathematics and Statistics, Xuzhou Institute of Engineering, Xuzhou, China
}

\begin{abstract}
China's national willingness to have children has been decreasing, according to the 2020 census, China's aging coefficient has been as high as $13.32 \%$, the very serious aging situation will have a wide impact on China's economic restructuring, transformation of production methods, social security system and so on. In this paper, we apply the requests library in python to crawl the population data published by the National Bureau of Statistics, and use MATLAB to construct four single models, logistic regression prediction, modified gray prediction, leslie model and fitting model, and then construct a combined model to predict the aging trend in China in the next five years, and finally give the following suggestions: first, adhere to family planning and further optimize the fertility policy; second, improve the social security system, deepen the reform of the pension system, and establish a pension system covering urban and rural areas; third, vigorously develop the aging industry.
\end{abstract}

Keywords. China's aging, portfolio model.

\section{Introduction}

According to the United Nations International Population Association, an area is said to be an older society when the proportion of people aged 65 (or 60) and above in the total population (called the aging coefficient) exceeds 7\% (or 10\%) [1]. According to China's census data, China's aging coefficient was $6.96 \%$ in 2000 and was about to enter an aging society, and by 2020 , the seventh census data showed that China's aging coefficient was already as high as $13.32 \%$, an increase of 4.79 percentage points compared to 2010 .

Against the backdrop of China's declining national fertility intentions and declining birth rates in general, the very serious ageing situation will have a wide impact on China's economic restructuring, transformation of production methods and social security system. Therefore, accurate prediction of the trend of the aging coefficient in China can provide reference data for the formulation of coordinated and sustainable development of population and economy, society, resources and environment in China.

Combinatorial forecasting models are models that combine multiple models in some way that combine the strengths of each individual forecasting model to make better forecasts of the data. Because although a single mathematical model can predict better, different models have their own advantages and disadvantages in prediction. In order to

\footnotetext{
${ }^{1}$ Corresponding author: Youhui SU, College of Mathematics and Statistics, Xuzhou Institute of Engineering, Xuzhou, China; E.mail: suyh02@163.com.
} 
give full play to the advantages of a single model in forecasting and reduce its disadvantages, each single model is given a certain weight through certain criteria and then a combined forecasting model is constructed. The combined forecasting model has been the focus of research by scholars at home and abroad, and scholars have explored the applicability of the model for different problems and proved that the combined forecasting model is indeed better than the single model in terms of forecasting accuracy $[2][3][4][5]$.

Therefore, in order to make the prediction more accurate, this paper introduces a combined prediction model to analyze the prediction. This paper uses MATLAB to construct four single models, logistic regression forecasting, modified gray forecasting, leslie model and fitting model, crawl China's population data from 1990 to 2020 through python and use it as a sample to find out the sum of squares of errors within the sample respectively, further determine the weights of each single model through the error square and derivative method to construct Combined model of population aging. Through the constructed combined prediction model, the level of aging in China from 2021 to 2025 is predicted, and the prediction results are derived and corresponding recommendations are given.

\section{Data Collection}

The State Bureau of Statistics is the department directly under the State Council that unifies the leadership of the national economy, culture and education, social and demographic surveys and statistics, is in charge of national statistics and national economic accounting, draws up regulations on statistical work, plans for statistical reform and statistical modernization, and national statistical survey plans, organizes and supervises the leadership and inspection of statistics and national economic accounting work in all regions and departments, and supervises and inspects the implementation of statistical laws and regulations. It is the official and authoritative statistical agency and source of data in China.

As the National Statistics Office in the census itself provides data on the site, there is a download interface available, according to the study found that although the data itself are encrypted can not be copied directly, but can be converted into excel reports for download, so we need to crawl the census page to all download addresses, and then a unified download.

This paper uses the requests library to crawl web pages. requests is an http library based on urlib and written in Python language. requests is more convenient than urllib, it can send requests to the web pages to be crawled, then automatically crawl html pages and simulate browser requests to submit, thus get the target web resource.

First determine that it is a static multi-template page, so only need to get the request address from the interface. Define tool functions to make requests, then import the requests package to simulate browser requests to prevent crawling, fill in the request source Rferer and browser type User-Agent, then decode the content, use content.decode to decode to jbk encoding, create html objects and convert to $\mathrm{xml}$ format, then import the package etree, use the xpath to extract the data. Finally, a function save is declared above to request the excel download address and save it to the index file in the same path of the code. After run, it starts to automatically crawl the data download address in the web page and automatically downloads it to the index folder in the form of excel for 
subsequent mathematical modeling analysis. The specific code is shown in the appendix and some of the crawling results are shown in tables 1,2 and 3:

Table 1. Number of households, population and sex ratio by district.

\begin{tabular}{|c|c|c|c|c|c|}
\hline \multirow[b]{2}{*}{ region } & \multicolumn{2}{|c|}{$\begin{array}{c}\text { household } \\
\end{array}$} & \multirow[b]{2}{*}{$\ldots$} & \multirow[b]{2}{*}{ sex ratio } & \multirow{2}{*}{$\begin{array}{c}\text { Average } \\
\text { household } \\
\text { size } \\
\end{array}$} \\
\hline & $\begin{array}{c}\text { add up the } \\
\text { total }\end{array}$ & $\begin{array}{c}\text { family } \\
\text { household }\end{array}$ & & & \\
\hline $\begin{array}{l}\text { national } \\
\text { Beijing, } \\
\text { capital of }\end{array}$ & 417722698 & 401934196 & $\cdots$ & 144.86 & 3.09 \\
\hline $\begin{array}{l}\text { People's } \\
\text { Republic of } \\
\text { China }\end{array}$ & 7355291 & 6680552 & $\ldots$ & 153.88 & 2.45 \\
\hline $\begin{array}{c}\text { Tianjin } \\
:\end{array}$ & $\begin{array}{c}3963604 \\
:\end{array}$ & $\begin{array}{c}3661992 \\
:\end{array}$ & $\begin{array}{l}\cdots \\
\ldots\end{array}$ & $\begin{array}{c}197.71 \\
:\end{array}$ & $\begin{array}{l}2.80 \\
: \vdots\end{array}$ \\
\hline Qinghai & 1586635 & 1529039 & $\ldots$ & 228.50 & 3.46 \\
\hline Ningxia & 1945064 & 1882205 & $\ldots$ & 177.09 & 3.17 \\
\hline Xinjiang & 6902850 & 6705607 & $\ldots$ & 178.51 & 3.10 \\
\hline
\end{tabular}

Table 2. Population by Age and Sex by Region.

\begin{tabular}{|c|c|c|c|c|c|}
\hline \multirow{2}{*}{ region } & \multicolumn{2}{|c|}{0 years } & \multirow[b]{2}{*}{$\cdots$} & \multicolumn{2}{|c|}{ Over 100 years old } \\
\hline & male & women & & male & women \\
\hline national & 7461199 & 6325235 & $\ldots$ & 8852 & 27082 \\
\hline Beijing, & 60564 & 55318 & $\ldots$ & 146 & 406 \\
\hline Tianjin & 43546 & 38325 & $\cdots$ & 80 & 136 \\
\hline$\vdots$ & $\vdots$ & $\vdots$ & $\cdots$ & $\vdots$ & $\vdots$ \\
\hline Qinghai & 37911 & 33753 & $\cdots$ & 32 & 52 \\
\hline Ningxia & 40062 & 35215 & $\cdots$ & 20 & 56 \\
\hline Xinjiang & 164879 & 155521 & $\ldots$ & 359 & 384 \\
\hline
\end{tabular}

Table 3. National fertility status of women of childbearing age by age and number of children.

\begin{tabular}{|c|c|c|c|c|c|}
\hline \multirow[b]{2}{*}{ age } & \multirow[b]{2}{*}{$\begin{array}{c}\text { Number of } \\
\text { births }\end{array}$} & \multirow[b]{2}{*}{ fertility } & \multirow[b]{2}{*}{$\cdots$} & \multicolumn{2}{|c|}{ Third child and above } \\
\hline & & & & $\begin{array}{c}\text { Number of } \\
\text { births }\end{array}$ & fertility \\
\hline aggregate & 1190060 & 33.31 & $\ldots$ & 77899 & 2.18 \\
\hline 15 & 89 & 0.11 & $\ldots$ & 0 & 0 \\
\hline 16 & 745 & 0.86 & $\ldots$ & 1 & 0 \\
\hline$\vdots$ & $\vdots$ & $\vdots$ & $\cdots$ & $\vdots$ & $\vdots$ \\
\hline 47 & 5526 & 4.93 & $\ldots$ & 1082 & 0.96 \\
\hline 48 & 4180 & 5.53 & $\ldots$ & 908 & 1.20 \\
\hline 49 & 2292 & 3.72 & $\ldots$ & 520 & 0.84 \\
\hline
\end{tabular}




\section{Modeling}

\subsection{Portfolio Prediction Modeling}

Combinatorial forecasting models are models that combine multiple models in some way that combine the strengths of each individual forecasting model to make better forecasts of the data. Because although a single mathematical model can predict better, different models have their own advantages and disadvantages in prediction. In order to give full play to the advantages of a single model in prediction and to reduce its disadvantages, each single model is given certain weights through certain criteria and later a combined prediction model is constructed [6].

In this paper, four models, least squares fitting, logistic model, modified $\operatorname{GM}(1,1)$ model and leslie model, are used as single models, and then weights are assigned by certain criteria to finally form a combined model to forecast the future population .

\subsection{Least Squares Fitting}

For data points with unknown relationships, the fitting method is often used to seek the relationship among them, and the least squares method is generally used to determine the values of the coefficients in the function, and the least squares method mainly uses the idea of function approximation, so that the residual sum of squares between the fitted function and the data points is minimized, then we have:

$$
\left.\sum_{i=1}^{N}\left[y_{i}-f(x)\right]^{2}\right|_{a=\hat{a}}
$$

where $f(x)$ is the point value of the fitted function and $y_{i}$ is the data in the original data column. The fitting was done using the fitting function toolbox in matlab to obtain the relationship between the population ageing index and year as follows:

$$
y=0.000001426 x^{5}+0.0001017 x^{4}-0.002852 x^{3}+0.03378 x^{2}+0.2781 x+5
$$

Plotting the image of the fitted function versus the data points gives: 


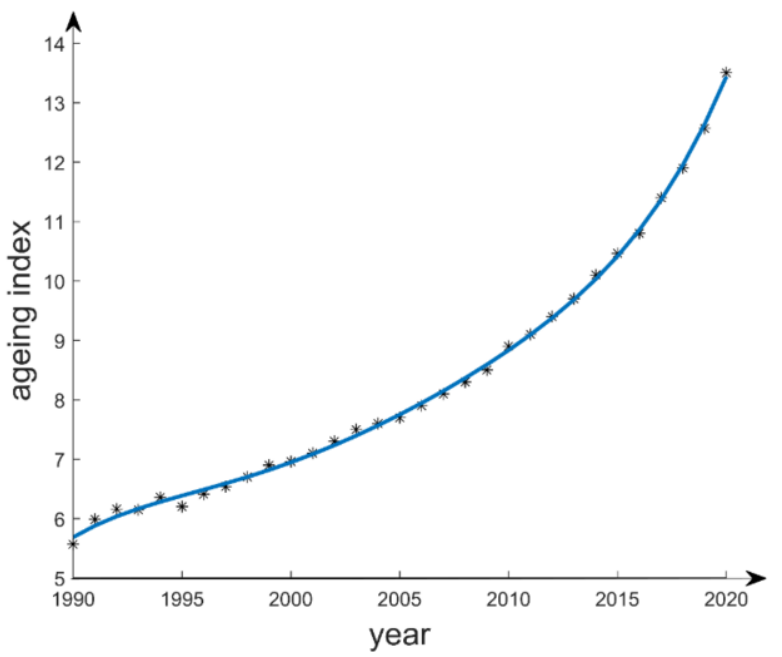

Figure 1. Aging Index Fitted Graph.

As can be seen from figure 1, with the growth of the year, the aging index grows simultaneously, and the rate of growth gradually increases, which to a certain extent reflects the urgent need to solve the aging problem in China. The data points basically satisfy the fitted curve, and it can be intuitively seen from the above figure that most of the data points are on the curve, and only a small number of data points deviate from the fitted curve, which indicates a good fitting effect, and in order to intuitively get the error value of the fitting effect, this paper treats the data with relative error and absolute error.

The accuracy of the model is then tested by $x=1990,1991, \cdots, 2020$ substituting into the above equation to obtain the fitted index of population ageing for 1990-2020, and comparing the fitted results with the actual values to calculate the relative error, which is shown in the table 4 below:

Table 4. Least squares fit error values.

\begin{tabular}{ccccc}
\hline year & fitted value & actual value & absolute error & $\begin{array}{c}\text { Relative error } \\
(\%)\end{array}$ \\
\hline 1990 & 5.69 & 5.57 & 0.12 & 2.09 \\
1991 & 5.88 & 5.99 & -0.11 & -1.82 \\
1992 & 6.04 & 6.16 & -0.12 & -1.98 \\
$\vdots$ & $\vdots$ & $\vdots$ & $\vdots$ & $\vdots$ \\
2018 & 11.97 & 11.90 & 0.07 & 0.60 \\
2019 & 12.66 & 12.57 & 0.09 & 0.70 \\
2020 & 13.03 & 13.50 & -0.48 & -3.54 \\
\hline
\end{tabular}

From the above table, the error that exists between the fitted and actual values is small, indicating a good fit, and this model can be used for the next step of combined forecasting, using the fitted functional relationship equation for the next five years, to obtain the forecast values in the following table: 
Table 5. Predicted Values from Least Squares Fitting.

\begin{tabular}{cccccc}
\hline year & 2021 & 2022 & 2023 & 2024 & 2025 \\
\hline $\begin{array}{c}\text { predicted } \\
\text { value }\end{array}$ & 13.9713 & 15.0821 & 16.3863 & 17.9151 & 19.7035 \\
\hline
\end{tabular}

From the above table 5, the aging projection for the next five years is $19.7035 \%$ by 2025 , which will have a large impact on the development of the country if the aging problem is not controlled.

\subsection{Logistic Growth Curve Model Estimation}

Logistic regression models are often used for data that fit a growth pattern, i.e., a data point set that grows exponentially in the previous period and contains a certain hysteresis coefficient. In order to find the trend in population size over time for a given region, calculus is often used to find the pattern, and the logistic growth curve model is obtained using the ordinary differential equation, which gives:

$$
\frac{d x}{d t}=r x\left(1-\frac{x}{x_{m}}\right)
$$

where $x$ is the original data column, $t$ is time, $x_{m}$ is the maximum population capacity, and $r$ is the annual population growth rate. The above equation is simplified using the separation of variables method:

$$
x(t)=\frac{x_{m}}{1+\left(\frac{x_{m}}{x_{0}}-1\right) e^{-r t}}
$$

where $r, x_{m}, x_{0}$ are the parameters, which are commonly estimated by least squares, numerical approximation, curve fitting, etc. In this paper, numerical differentiation is used to obtain the logistic growth curve equation:

$$
x(t)=\frac{1}{0.188 \times 0.974^{t}}
$$

The model equation above was calculated using SPSS for the last 30 years and the goodness of fit of the logistic growth curve equation was obtained as 0.983 , indicating a good fit of the model to the data, and the relationship between the logistic fit curve, the primary function fit results and the original data points is shown in the following figure: 


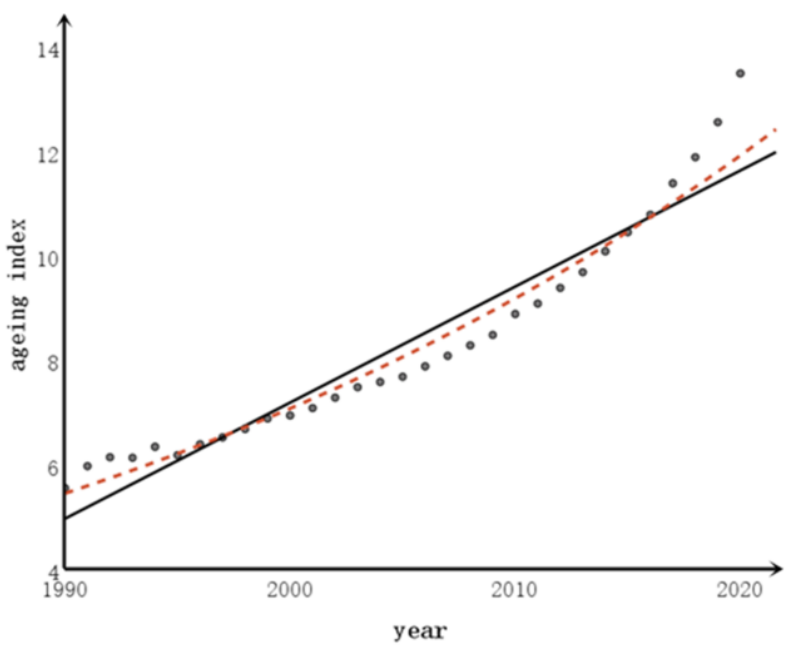

Figure 2. Logistic function fit graph.

From the above figure 2, the trajectory composed of dots is the original data point set, and the solid line is the fitting result of the primary function, which basically conforms to the growth trend of the aging index with the year, but there is still a large error for accurate prediction; comparing the fitted curve by Logistic, its fitted data points in 1990-2010 are more in line with the actual value, and the error between the fitted value and the actual value is larger in the latter years.

Considering that the logistic growth curve model fits and predicts the function according to the general growth law, and there are many factors that affect the fluctuating changes of the population aging index, such as: national policies, population migration rate, the spread of disease epidemics, etc., its impossible to get accurate prediction values. However, the logistic growth curve model ideally depicts the growth pattern to a certain extent, and has some reference significance for the prediction results.

The error between the estimated and actual values is obtained so that the accuracy of the function can be evaluated, and the error between the two is shown below.

Table 6. Logistic error values.

\begin{tabular}{ccccc}
\hline year & $\begin{array}{c}\text { predicted } \\
\text { value }\end{array}$ & actual value & absolute error & $\begin{array}{c}\text { Relative error } \\
(\%)\end{array}$ \\
\hline 1990 & 5.47 & 5.57 & -0.10 & -1.80 \\
1991 & 5.61 & 5.99 & -0.38 & -6.29 \\
1992 & 5.76 & 6.16 & -0.40 & -6.48 \\
$\vdots$ & $\vdots$ & $\vdots$ & $\vdots$ & $\vdots$ \\
2018 & 11.33 & 11.90 & -0.57 & -4.81 \\
2019 & 11.63 & 12.57 & -0.94 & -7.51 \\
2020 & 11.93 & 13.50 & -1.57 & -11.64 \\
\hline
\end{tabular}

From the above table 6 , the error between the predicted and actual values is large, and the relative error of the prediction for 2020 reaches $11.64 \%$, which is a large error, but the overall error is small, and the model satisfies the general growth law, but does not take into account the influence of other factors, and the prediction is average. 
Using the model developed to estimate the ageing index for the next five years, we have table 7:

Table 7. Logistic Prediction Values

\begin{tabular}{cccccc}
\hline year & 2021 & 2022 & 2023 & 2024 & 2025 \\
\hline $\begin{array}{c}\text { predicted } \\
\text { value }\end{array}$ & 12.2466 & 12.5692 & 12.9004 & 13.2402 & 13.5890 \\
\hline
\end{tabular}

Compared to the results of the least squares fit, the logistic growth curve model predicts a smaller increase, which is more in line with the general pattern of the "S" curve in the growth law.

\subsection{Modified Gm(1,1) Projections}

$\operatorname{GM}(1,1)$ is a prediction method for gray systems, which is generally more accurate for small samples. gray prediction is to find the pattern of system changes by judging the degree of dissimilarity of development trends between system factors, i.e., correlation analysis of two factors, and generating processing of the original data to generate a data series with strong regularity, and then establish a differential equation model, so as to predict the future of things The whitening equation of $\operatorname{GM}(1,1)$ model is:

$$
\frac{d x^{(1)}}{d t}+a x^{(1)}=b
$$

where the time response function is:

$$
x^{(1)}(k+1)=\left(x^{(1)}(1)-\frac{b}{a}\right) e^{(-a k)}+\frac{b}{a}
$$

where $x^{(1)}(k)$ is the series after one accumulation, $a$ is the development factor, and $b$ is the amount of gray action by $\left[\frac{a}{b}\right]=\left(B^{T} * B\right)^{-1} B^{T} Y$ determining the values of $a, b$. Where $B$ and $Y$ are matrices and vectors composed of the original data with the expressions:

$$
B=\left[\begin{array}{cc}
-\frac{1}{2}\left(z^{(1)}(1)+z^{(1)}(2)\right) & 1 \\
-\frac{1}{2}\left(z^{(1)}(2)+z^{(1)}(3)\right) & 1 \\
\vdots & \vdots \\
-\frac{1}{2}\left(z^{(1)}(n-1)+z^{(1)}(n)\right) & 1
\end{array}\right], \quad Y=\left[\begin{array}{c}
x^{(0)}(2) \\
x^{(0)}(3) \\
\vdots \\
x^{(0)}(n)
\end{array}\right]
$$


Substituting the original data to perform the calculation gives the matrix $B$ and the column vector $Y$. Then we have:

$$
B=\left[\begin{array}{cc}
-5.6328 & 1 \\
-5.8685 & 1 \\
\vdots & \vdots \\
-12.1009 & 1
\end{array}\right], Y=\left[\begin{array}{c}
5.9902 \\
6.1602 \\
\vdots \\
13.5035
\end{array}\right]
$$

Calculations were performed using the matrix to obtain parameter values for the development factor $a$ and the amount of grey action $b$ :

$$
\hat{a}=\left(B^{T} * B\right)^{-1} B^{T} Y=\left[\begin{array}{c}
-0.0283 \\
5.1612
\end{array}\right]
$$

Substituting the parameters into the time response function, we have:

$$
x^{(1)}(k+1)=\left(x^{(1)}(1)-\frac{b}{a}\right) e^{(-a k)}+\frac{b}{a}=187.94 e^{0.0283 t}-182.37
$$

Different values of $t$ were taken, and the model was solved and error analyzed using Matlab to obtain the following error values for the aging index for 1990-2020:

Table 8. Gray prediction error values.

\begin{tabular}{ccccc}
\hline year & $\begin{array}{c}\text { predicted } \\
\text { value }\end{array}$ & actual value & absolute error & $\begin{array}{c}\text { Relative error } \\
(\%)\end{array}$ \\
\hline 1990 & 5.5687 & 5.57 & -0.0010 & -0.02 \\
1991 & 5.985442 & 5.99 & -0.005 & -0.08 \\
1992 & 6.146646 & 6.16 & -0.01 & -0.22 \\
$\vdots$ & $\vdots$ & $\vdots$ & $\vdots$ & $\vdots$ \\
2018 & 11.67 & 11.90 & -0.23 & -1.91 \\
2019 & 12.76 & 12.57 & 0.19 & 1.54 \\
2020 & 13.29 & 13.50 & -0.21 & -1.56 \\
\hline
\end{tabular}

From the above table 8 , the error between the corrected grey prediction after and the actual value is less, the relative error of the uncorrected grey prediction is $5.7 \%$ on average, while the relative error of the corrected grey prediction is $1.4 \%$ on average, which indicates that the accuracy of this model is better and this model can be used for the next combination of prediction.

Using the model to project the ageing index for the next five years, we have table 9:

Table 9. Grey Forecast Predicted Values.

\begin{tabular}{cccccc}
\hline year & 2021 & 2022 & 2023 & 2024 & 2025 \\
\hline $\begin{array}{c}\text { predicted } \\
\text { value }\end{array}$ & 12.6046 & 12.9662 & 13.3383 & 13.7210 & 14.1146 \\
\hline
\end{tabular}




\subsection{Leslie Projection Population Model}

For the general population projection model, only the total number of people is projected, leslie projection population model divides the population into groups according to age groups and makes projections based on parameters such as population migration rate and fertility rate, which have practical implications for the projection of the number of people in the age groups. Its expressions are:

$$
x_{j}^{l}(i+1, k+1)=s_{j}^{l}(i, k) x_{j}^{l}(i, k)+v_{j}^{l}(i, k)
$$

where $x_{j}^{l}(i+1, k+1)$ is the evolution of the future population size, $s_{j}^{l}(i, k)$ is the population survival rate, and $v_{j}^{l}(i, k)$ is the number of displaced persons. The ratio of the number of births to women of reproductive age at age is:

$$
h_{j}^{w}(i, k)=\frac{b_{j}^{w}(i, k)}{\beta_{j}^{w}(k)}
$$

where $h_{j}^{w}(i, k)$ satisfies $\sum_{i=i_{1}}^{i_{2}} h_{j}^{w}(i, k)=1$, with age as the discrete variable, and divides the data by gender to obtain the set of difference equations:

$$
\left\{\begin{array}{c}
x_{1}^{m}(k+1)=S_{1}^{m}(k) x_{1}^{m}(k)+s_{1}^{m}(0, k) a_{1}(k) \beta_{1}^{w}(k) H_{1}^{w}(k) x_{1}^{w}(k)+v^{m}(k) \\
x_{1}^{w}(k+1)=S_{1}^{w}(k) x_{1}^{w}(k)+s_{1}^{w}(0, k)\left(1-a_{1}(k)\right) \beta_{1}^{w}(k) H_{1}^{w}(k) x_{1}^{w}(k)+v^{w}(k) \\
\vdots \\
x_{n}^{w}(k+1)=S_{n}^{w}(k) x_{n}^{w}(k)+S_{n}^{w}(0, k)\left(1-a_{n}(k)\right) \beta_{n}^{w}(k) H_{n}^{w}(k) x_{n}^{w}(k)+v^{w}(k)
\end{array}\right.
$$

Parameter estimates of the survival rate, the sex ratio of infants, and the number of population migrations are used to project the ageing index for the next five years, with the following error values for the ageing index for 1990-2020:

Table 10. leslie model error values.

\begin{tabular}{ccccc}
\hline year & predicted value & actual value & absolute error & $\begin{array}{c}\text { Relative error } \\
(\%)\end{array}$ \\
\hline $\mathbf{1 9 9 0}$ & 5.67 & 5.57 & 0.10 & 1.80 \\
$\mathbf{1 9 9 1}$ & 5.85 & 5.99 & -0.14 & -2.34 \\
$\mathbf{1 9 9 2}$ & 5.98 & 6.16 & -0.18 & -2.93 \\
$\vdots$ & $\vdots$ & $\vdots$ & $\vdots$ & $\vdots$ \\
$\mathbf{2 0 1 8}$ & 11.70 & 11.90 & -0.20 & -1.68 \\
$\mathbf{2 0 1 9}$ & 12.20 & 12.57 & -0.37 & -2.95 \\
$\mathbf{2 0 2 0}$ & 12.70 & 13.50 & -0.80 & -5.95 \\
\hline
\end{tabular}


From the above table 10, the error between the predicted and actual values of leslie model is small and the relative error is stable at about $2 \%$, which indicates that the model works well and this model can be used for the next combination of prediction.

Using the above model to project the ageing index for 2021-2025, we have table 11:

Table 11. leslie model predicted values.

\begin{tabular}{cccccc}
\hline year & 2021 & 2022 & 2023 & 2024 & 2025 \\
\hline $\begin{array}{c}\text { predicted } \\
\text { value }\end{array}$ & 13.7 & 14.4 & 14.8 & 15.0 & 16.2 \\
\hline
\end{tabular}

The leslie model is solved and predicted based on multiple parameters such as birth rate and population migration rate, and to a certain extent the prediction results are more in line with the reality.

\section{Projections of the Ageing Index}

The combined forecasting model combines multiple forecasting methods and obtains the final forecast value by weighting method, which combines the advantages of each forecasting method and reduces the error generated by a single forecasting model to a certain extent.

The least squares fit, grey $\operatorname{GM}(1,1)$ model, leslie population projection model, and logistic growth curve were used above to estimate and predict the population aging index, respectively. In this paper, the results obtained from the four methods are assigned weights using the error sum of squares method to obtain the predicted values after the combined consideration.

In this paper, the predictive performance of the method is evaluated based on the magnitude of the sum of squares of errors, which is the sum of squares of the differences between the estimated and actual values of each sample prediction, and the larger the value, the worse the prediction of the method, and the formula for calculating the sum of squares of errors is as follows:

$$
E_{j}=\sum_{i=1}^{30}\left(\hat{x}_{i, j}-x_{i, j}\right)^{2}
$$

where $E_{j}(j=1,2,3,4)$ denotes the jth method, $\hat{x}_{i, j}$ denotes the predicted value of the $j$-th method for the $i$-th sample, and $x_{i, j}$ denotes the actual value of the $j$-th method for the $i$-th sample. The degree of superiority of each method is obtained by comparing the magnitude of the sum of squares of the errors, and we have:

Table 12. Ranking of individual model prediction effects.

\begin{tabular}{ccc}
\hline approach & Error sum of squares & sorted \\
\hline Grey GM(1,1) model & 1.67 & 3 \\
leslie model & 1.6145 & 2 \\
Logistic curve estimation & 5.6918 & 4 \\
least-squares fit & 0.3897 & 1 \\
\hline
\end{tabular}


From the above table 12, the best prediction result is least squares fitting and the worst is logistic curve estimation. The sum of squared errors is negativized, i.e., the inverse of the sum of squared errors is found to obtain the weights of each prediction method, and the final prediction results are obtained according to the magnitude of the weights. The final prediction result formula is as follows:

$$
K=w_{1} \hat{x}_{1}+w_{2} \hat{x}_{2}+w_{3} \hat{x}_{3}+w_{4} \hat{x}_{4}
$$

where $w_{j}(j=1,2,3,4)$ denotes the weight of each method obtained from the sum of squared errors, and $\hat{x}_{j}(j=1,2,3,4)$ denotes the result obtained from the prediction of each method.

Using the above equation to obtain the weights of each method in the following order: $0.1557,0.1612,0.1341$ and 0.5941 , the population ageing index for the next five years can be predicted as follows in table 13:

Table 13. Combined prediction model results.

\begin{tabular}{cccccc}
\hline year & 2021 & 2022 & 2023 & 2024 & 2025 \\
\hline Ageing index & 14.0282 & 14.9102 & 15.8500 & 16.9155 & 18.2431 \\
\hline
\end{tabular}

The data were plotted as a bar chart as follows:

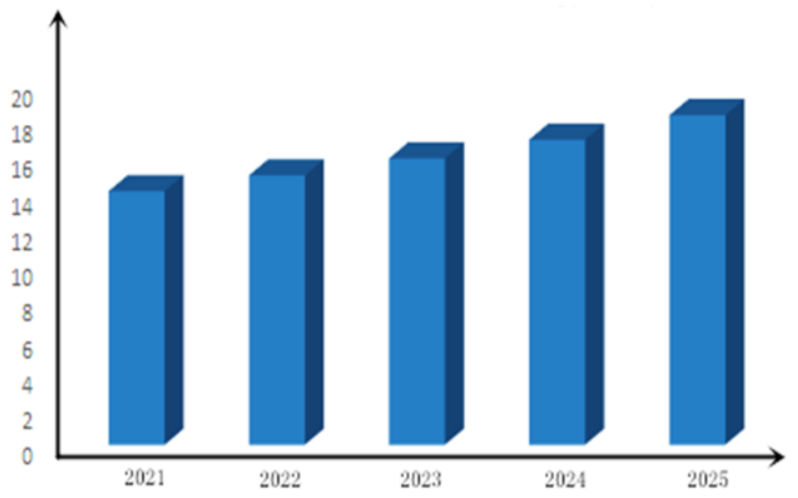

Figure 3. Projected Ageing Index 2021-2025.

From the above figure 3, as the years grow, the aging index grows, and according to the current growth trend, by 2025 , the aging index will be $18.3372 \%$, and the critical value of aging society defined by the World Health Organization is $10 \%$, and the predicted value in 2025 is nearly twice the critical value, and the result will seriously affect urbanization construction, labor supply level, etc. Therefore, solving the The problem of population ageing cannot be delayed.

\section{Conclusions and Recommendations}

In this paper, we first use python to crawl census data from the National Bureau of Statistics (NBS) and annual population sample survey data as sample data through the requests and etree packages, and then construct four single models for predicting 
population aging by least squares fitting, logistic model, modified $\operatorname{GM}(1,1)$ model, and leslie model, respectively.

The prediction errors of each individual model within the sample were calculated from the sample data, followed by the method of the sum of squares of the difference between each sample prediction estimate and the actual value, i.e., the sum of squares of errors, and finally the sum of squares of errors was negativized, i.e., the inverse of the sum of squares of errors, and the weights of each prediction method were obtained in the following order: $0.1557,0.1612,0.1341$, and 0.5941 , and according to the weight of size, the final projections of population aging in the next five years are obtained as 14.0282, $14.9102,15.8500,16.9155$ and 18.2431 in order.

Forecast data show that in the next five years, the country's ageing index will continue to rise and ageing will become more serious, and the following recommendations are made in the light of our basic national conditions:

(1) The ageing of the population has led to a decline in the proportion of the working-age population. Family planning is being adhered to, and fertility policies are being further optimized, with the implementation of the policy of allowing a couple to have three children and supporting measures to improve the country's demographic structure, implement the national strategy to actively respond to population ageing and maintain the advantages of our human resource endowment.

(2) The burden of old-age security is becoming heavier and heavier, and the pressure on consumer spending on medical and health care for the elderly is increasing. It is improving the social security system, deepening the reform of the pension system, and establishing a pension system covering both urban and rural areas.

(3) The demand for social services for the elderly is expanding rapidly, and the market for the elderly is promising. As most of the elderly industry belongs to the tertiary sector, promoting the development of the elderly industry will help to promote the optimization and upgrading of China's industrial structure, meet the needs of the elderly population while reducing the burden of old age on young people, provide employment and provide a new impetus for China's economic development.

\section{Acknowledgments}

Fund Project: Jiangsu University Student Innovation Program (xcx2021190)

\section{References}

[1] Chen Weijie and Lv Shengige. Medium- and long-term projections of population aging coefficient in Sichuan Province[J]. Statistics and Decision Making,2013(01):125-128.

[2] Xiong Wei,Qi Chuncheng,Gao Yu and Yang Chun. Research on short-term forecasting of agricultural market prices based on combinatorial modeling--Red Fuji apples, bananas and oranges as examples[J]. Agricultural Technology Economics,2015(06):57-65.

[3] Song Xiaohua,Zu Pi'e,Yi Jing and Liu Da. A long-term power optimization portfolio forecasting model based on improved $\operatorname{GM}(1,1)$ and SVM[J]. Journal of Central South University (Natural Science Edition),2012,43(05):1803-1807.

[4] David E. Rapach and Jack K. Strauss. Structural breaks and GARCH models of exchange rate volatility[J]. Journal of Applied Econometrics,2008,23(1):

[5] Sumru Altug and Cem Çakmaklı. forecasting inflation using survey expectations and targetinflation:evidence for Brazil and Turkey[J]. International Journal of Forecasting,2016,32(1):

[6] Bates, J.M. and Granger, C.W.J. (2001) The Combination of Forecasts. Cambridge University Press, Cambridge, 451-468. 


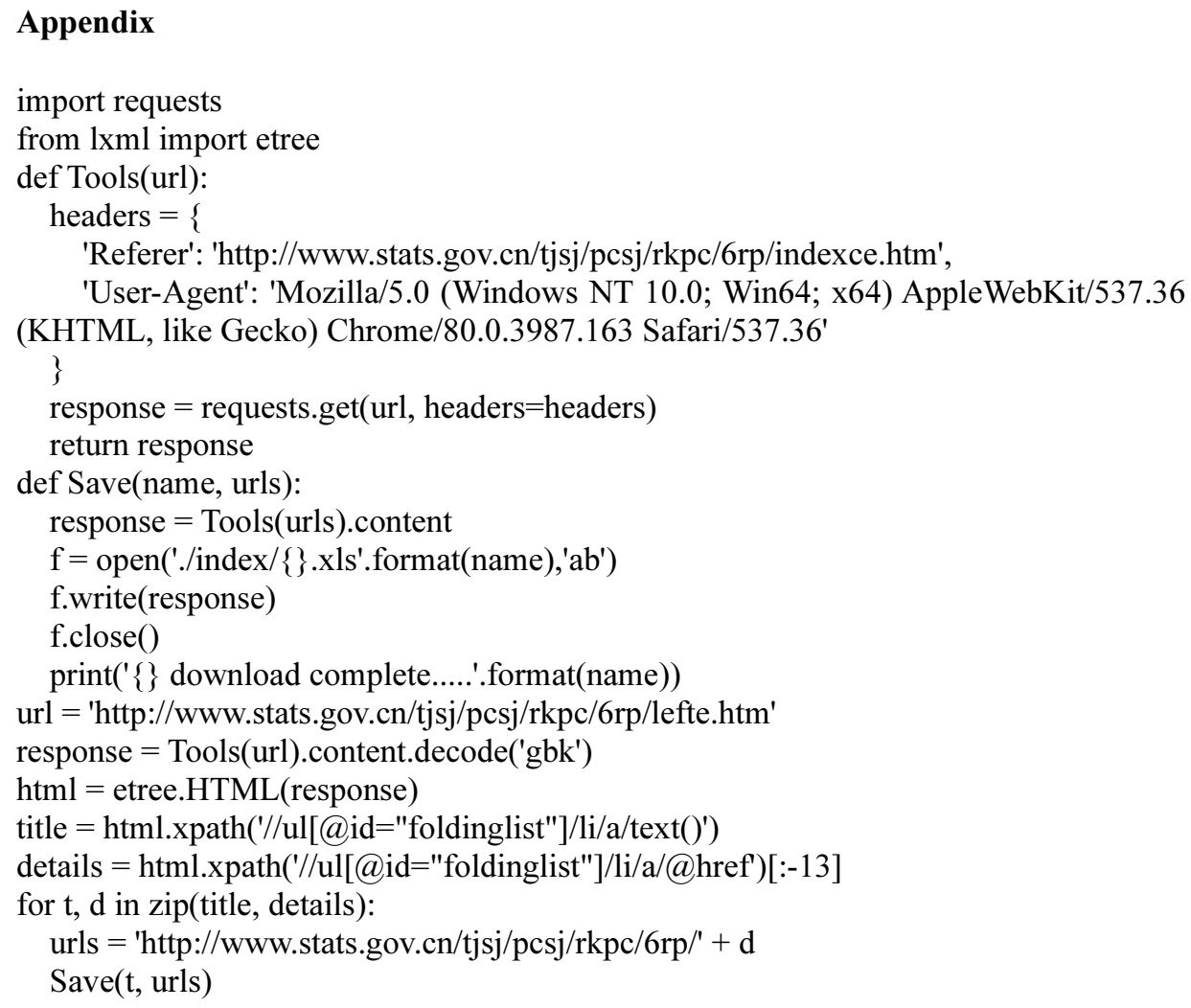

